\title{
ON THE FRACTIONAL DIFFERENTIATION OF A FUNCTION OF SEVERAL VARIABLES
}

\author{
BY \\ G. V. WELLAND
}

1. In [5] a characterization for fractional differentiation of a function of a real variable is given. Here, the results are extended to the case of a function of several variables.

Before we state these results we must review some definitions. By $x, y, t, \ldots$ we denote points $\left(x_{1}, x_{2}, \ldots, x_{n}\right),\left(y_{1}, y_{2}, \ldots, y_{n}\right), \ldots$ of $n$-dimensional Euclidean space. We will consider integrable functions, $f: E_{n} \rightarrow R$, where $R$ is the set of real numbers. We denote the measure of a measurable set $E$ by $|E|$. The symbols $x+y$ and $\lambda x$, where $\lambda$ is a scalar, have the usual meaning. By $|x|$ we mean $\left(\sum_{i=1}^{n} x_{i}^{2}\right)^{1 / 2}$, by $\langle x, y\rangle$ we mean $\sum_{i=1}^{n} x_{i} y_{i}$, and if $j=\left(j_{1}, j_{2}, \ldots, j_{n}\right)$ where the $j_{i}$ are integers we use the notation $|j|$ to mean $j_{1}+j_{2}+\cdots+j_{n}$. By $x^{j}$ we mean $x_{1}^{j_{1}} x_{2}^{j_{2}} \cdots x_{n}^{j_{n}} . \Sigma$ denotes the unit sphere of $E_{n}, \sigma, \mu$ denote elements of $\Sigma$, and $d \sigma$ the usual area element of $\Sigma$.

We will use the symbol $C$, sometimes with subscripts and sometimes without, for an absolute constant or a constant dependent only on the dimension and the parameters of the problem.

By $C_{0}^{k}$ we denote the class of functions with compact support and $k$ continuous derivatives, and we write $(\partial / \partial x)^{j}$ for $\left(\partial^{j_{1}} / \partial x_{1}^{j_{1}}\right)\left(\partial^{j_{2}} / \partial x_{2}^{j_{2}}\right) \cdots\left(\partial^{j_{n}} / \partial x_{n}^{j_{n}}\right)$ and $(\partial / \partial x)^{j} f=f_{j}$.

For a function $f$, we denote its $\beta$ th integral by $f_{\beta}$ and write

$$
f_{\beta}(x)=\int_{E_{n}} \frac{f(t) d t}{|x-t|^{n-\beta}} \quad(0<\beta<1) .
$$

Whenever the region of integration for an integral is $E_{n}$ we may simply write $\int$ for $\int_{E_{n}}$.

A function defined in a neighborhood of a point $x_{0}$ is said to have a $k$ derivative if

$$
f\left(x_{0}+t\right)=P_{x_{0}}(t)+R_{x_{0}}(t)
$$

where $P_{x_{0}}(t)$ is a polynomial in the variable $t$ of degree $\leqq k$ and $R(t)=R_{x_{0}}(t)$ $=O\left(|t|^{k}\right)$ as $|t| \rightarrow 0$. For $1 \leqq p<\infty, f$ is said to have a derivative of order $k$ in the $L^{p}$ sense if $\left\{\rho^{-n} \int_{|t| \leqq \rho}|R(t)|^{p} d t\right\}^{1 / p}=o\left(\rho^{k}\right)$ as $\rho \rightarrow 0$.

For an integer $k \geqq 0, f$ is said to have a derivative of order $\alpha$ at $x_{0}, k<\alpha<k+1$, if $f_{\beta}$ has a $k+1$ derivative at $x_{0}(\alpha+\beta=k+1)$. $f$ is said to have an $\alpha$ derivative at $x_{0}$ in the $L^{p}$ sense if $f_{\beta}$ has a $k+1$ derivative at $x_{0}$ in the $L^{p}$ sense.

Received by the editors March 30, 1967. 
Furthermore, with $R(t)$ defined as in (1), $f$ is said to satisfy $\Lambda_{\alpha}$ at $x_{0}$ if $R(t)$ $=O\left(|t|^{\alpha}\right)$ as $|t| \rightarrow 0$. In this case we write $f \in \Lambda_{\alpha}$. The corresponding $L^{p}$ definition, $\Lambda_{\alpha}^{p}$, is satisfied if

$$
\left\{\frac{1}{\rho^{n}} \int_{|t|<\rho}|R(t)|^{p} d t\right\}^{1 / p}=O\left(\rho^{\alpha}\right) .
$$

Finally $f$ is said to satisfy the condition $N_{\alpha}^{p}$ if

$$
\int_{|t| \leqq \rho} \frac{|R(t)|^{p}}{|t|^{n+p \alpha}} d t<\infty \quad \text { for some } \rho>0 .
$$

The main theorems are as follows.

THEOREM 1. Suppose $f$ satisfies the condition $\Lambda_{\alpha}$ at every point of a measurable set $E, E$ has positive measure. Then the necessary and sufficient condition for $f$ to have a derivative of order $\alpha$ almost everywhere in $E$ is that $f$ satisfy the condition $N_{\alpha}^{2}$ almost everywhere in $E$.

The condition $N_{\alpha}^{2}$ is the characterizing notion for the fractional differentiability of functions, and if $\Lambda_{\alpha}$ is relaxed to $\Lambda_{\alpha}^{2}$ we have:

THEOREM 2. $f$ satisfies the condition $N_{\alpha}^{2}$ almost everywhere in the set $E$ if and only if $f$ satisfies the condition $\Lambda_{\alpha}^{2}$ and $f$ has an $\alpha$ derivative in the $L^{2}$ sense almost everywhere in $E$.

That $N_{\alpha}^{2}$ is the characterizing notion for differentiability is perhaps underlined by:

THEOREM 2'. $f$ has an $\alpha$ derivative in the sense of $L^{p}(2 \leqq p<\infty)$ and satisfies the condition $\Lambda_{\alpha}^{p}$ almost everywhere in a set $E$ if and only if $f$ satisfies both $N_{\alpha}^{2}$ and $N_{\alpha}^{p}$ almost everywhere in the set $E$.

The proofs of these results rely heavily on some theorems first proved in [4] for the one-dimensional case and then recently extended to the $n$-dimensional case in [3]. To state these results we make some further remarks.

Suppose that $f$ has a $k-1$ derivative at $x_{0}$ in either the ordinary sense or the $L^{p}$ sense and the polynomial for this case as corresponds to (1) is $P_{x_{0}}(t)$. Write $\Delta_{x_{0}}^{k}(t)=R_{x_{0}}(t)+(-1)^{k-1} R_{x_{0}}(-t) . f$ is said to satisfy the condition $\Lambda_{k}^{*}$ at $x_{0}$ if $\Delta_{x_{0}}^{k}(t)=O\left\{|t|^{k}\right\}$ as $|t| \rightarrow 0$ and it is said to satisfy the condition $N_{k}^{p}$ if there is a $\rho>0$ such that

$$
\int_{|t| \leqq \rho} \frac{\left|\Delta_{x_{0}}^{p}(t)\right|^{p}}{|t|^{n+p k}} d t<\infty \quad(1 \leqq p<\infty) .
$$

The results we speak of are given below.

THEOREM A. Suppose $f$ satisfies the condition $\Lambda_{k}^{*}$ at every point of a set $E$. Then $f$ has a $k$ derivative at almost every $x$ in $E$ if and only if $f$ satisfies $N_{k}^{2}$ almost everywhere in $E$. 
THEOREM B. The necessary and sufficient condition for $f$ to have a $k$ derivative in the $L^{p}$ sense $(2 \leqq p<\infty)$ almost everywhere in a set $E$ is that $f$ satisfies the conditions $N_{k}^{p}, N_{k}^{2}$ almost everywhere in $E$.

Theorem B is not proved in [3] but this result can be obtained in much the same way as it is done in the one-dimensional case in [4].

The proof of Theorem $2^{\prime}$ does not differ from the proof of Theorem 2 and we will only fully prove Theorem 1 and Theorem 2 . The existence of the $\alpha$ derivative at $x_{0}$ of $f$ depends on its local properties and hence altering $f$ outside a neighborhood of $x_{0}$ does not change the existence of the $\alpha$ derivative.

2. In addition to the above results several lemmas are used in the proofs.

LEMMA 1. (See [2, p. 148] for a similar case.) Let $f(x, y)$ be a measurable function on the product of two measure spaces $M_{1} \times M_{2}$ with the measures $\mu_{1}$ and $\mu_{2}$ respectively. Then using the usual notation for $L^{p}$ norms, $1 \leqq p<\infty,\left\|\int f(\cdot, y) d \mu_{2}\right\|_{p}$ $\leqq \int\|f(\cdot, y)\|_{p} d \mu_{2}$ where the $L^{p}$ norm is taken in the variable $x$.

Lemma 2. Suppose that $k$ is an integer, $0 \leqq k<\alpha<k+1$, and $\alpha+\beta=k+1$. Let $g$ be defined by

$$
g(x)=\int_{E_{n}} \frac{G_{\sigma}(x+t)}{|t|^{n+\beta-1}} d t
$$

where $\sigma=t /|t|, G_{\sigma}$ is the direction derivative of $G$ in the direction of $\sigma$, and $G \in C_{0}^{k+1}$. Then $g$ satisfies the condition $\Lambda_{\alpha}$ uniformly and $g$ satisfies $N_{\alpha}^{2}$ and $N_{\alpha}^{p}$ for almost all $x$ in $E_{n}, 2 \leqq p<\infty$.

Proof. Let $\mu$ be a unit vector in $E_{n}$ and by $g_{\mu^{k}}(x)$ denote the direction derivative of $g$ of order $k$ in the direction of $\mu$. Consider

$$
\begin{aligned}
& g_{\mu^{k}}(x+\mu \tau)-g_{\mu^{k}}(x)= \Delta_{g}^{k}(x, \mu, \tau)=\int_{E_{n}} G_{\sigma, \mu^{k}}(x+t+\mu \tau)-G_{\sigma, \mu^{k}}(x+t) \frac{d t}{|t|^{n+\beta-1}} \\
&= \int_{\Sigma} d \sigma \int_{0}^{\infty}\left(G_{\sigma, \mu^{k}}(x+r \sigma+\tau \mu)-G_{\sigma, \mu^{k}}(x+r \sigma)\right) \frac{d r}{r^{\beta}} \\
&= \int_{\Sigma} d \sigma \int_{0}^{\infty}\left(G_{\sigma, \mu^{k}}(x+r \sigma+\tau \mu)-G_{\sigma, \mu^{k}}(x+\tau \sigma+r \sigma)\right) \frac{d r}{r^{\beta}} \\
& \quad+\int_{\Sigma} d \sigma \int_{0}^{\infty}\left(G_{\sigma, \mu^{k}}(x+\tau \sigma+r \sigma)-G_{\sigma, \mu^{k}}(x+r \sigma)\right) \frac{d r}{r^{\beta}} \\
&= I_{1}+I_{2} . \\
&\left|I_{2}\right|=\left|\int_{\Sigma} d \sigma\left[\int_{0}^{\tau} G_{\sigma, \mu^{k}}(x+s \sigma) \frac{d s}{s^{\beta}}+\int_{\tau}^{\infty} G_{\sigma, \mu^{k}}(x+s \sigma)\left\{\frac{1}{|s-\tau|^{\beta}}-\frac{1}{s^{\beta}}\right\} d s\right]\right| \\
& \leqq \\
&|\Sigma|\left\|G_{\sigma, \mu^{k}}\right\|_{\infty} \mid \int_{0}^{\infty} \frac{d s}{|s-\tau|^{\beta}-\left.s^{\beta}|\leqq C| \tau\right|^{1-\beta}} ; \\
&\left|I_{1}\right|=\int_{\Sigma} d \sigma\left\{\int_{\tau}^{\infty}+\int_{0}^{\tau} \Delta^{k} G_{\sigma}(x, \mu, \tau, r) \frac{d r}{r^{\beta}}\right\}
\end{aligned}
$$


where $\Delta^{k} G_{\sigma}=\Delta^{k} G_{\sigma}(x, \mu, \tau, r)$ is the integrand of $I_{1}$. The first of these integrals can be estimated by first integrating by parts and noting that $G_{\mu}{ }^{k}$ is Lipschitz. The second integral can be estimated by replacing $\Delta^{k} G_{\sigma}$ by its maximum and integrating. Hence $\left|I_{2}\right| \leqq C|\tau|^{1-\beta}$. It only remains to apply Taylor's theorem to $g$ to see that $g$ is in $\Lambda_{\alpha}$, with the polynomial in (1) for $g$ being its Taylor's development of order $k$.

Next, to show $g$ satisfies the condition $N_{\alpha}^{2}$, define the function $\omega_{x, \mu}(t)$ by

$$
\omega_{x, \mu}(t)|t|^{1-\beta}=\left|g_{\mu^{k}}(x+t)-g_{\mu^{k}}(x)\right|
$$

with $\mu=t /|t|$. We first show

$$
\int_{E_{n}} \frac{\left|\omega_{x, u}(t)\right|^{2}}{|t|^{n}} d t<\infty
$$

To do this integrate (4) with respect to $x$. After an application of Parseval's equality we have

(5) $\frac{1}{(2 \pi)^{n}} \int_{E_{n}}\left\{\int_{E_{n}} \frac{\left|\omega_{x, \mu}(t)\right|^{2}}{|t|^{n}} d t\right\} d x=\int_{E_{n}}|t|^{2(\beta-1)-n} d t \int_{E_{n}} 2 \sin ^{2} \frac{\langle y, t\rangle}{2}\left|g_{\mu^{k}}(y)\right|^{2} d y$ where $g_{\mu^{k}}(y)=\int_{E_{n}} g_{\mu^{k}}(x) \cdot e^{-i\langle x, y\rangle} d x$. The integral

$$
\int_{E_{n}}|t|^{2(\beta-1)-n}|y|^{2(1-\beta)} 2 \sin ^{2}(\langle y, t\rangle / 2) d t \leqq C
$$

where $C$ is a constant depending on only $\beta$ and $n$. The remaining integral is equal to the square of

$$
\begin{aligned}
\left\{\int_{E_{n}}\left[\int_{\Sigma} d \sigma \int_{0}^{\infty} e^{-i\langle y, r \sigma\rangle} G_{\sigma, \mu^{k}}(y) \cdot|y|^{\beta-1} \frac{d r}{r^{\beta}}\right]^{2} d y\right\}^{1 / 2} & \\
= & \left\{\int_{E_{n}}\left[\int_{\Sigma} G_{\sigma, \mu^{k}}(y) \cdot|y|^{\beta-1} d \sigma \int_{0}^{\infty} e^{-i\langle y, r \sigma\rangle} \frac{d r}{r^{\beta}}\right]^{2} d y\right\}^{1 / 2} .
\end{aligned}
$$

But $\left|\int_{0}^{\infty} e^{-i\langle y, r \sigma\rangle} r^{-\beta} d r\right|=$ constant $\cdot|\langle y, \sigma\rangle|^{1-\beta}<C|y|^{1-\beta}$. Using this and applying Lemma 1 we have (5) is $\leqq C \int_{\Sigma}\left\{\int_{E_{n}}\left|G_{\sigma, \mu^{k}}(y)\right|^{2} d y\right\}^{1 / 2} d \sigma \leqq C|\Sigma|\left\|G_{\sigma, \mu^{k}}\right\|_{2}<\infty$.

Now let $x_{0}$ be a point where (4) is finite. Writing $g\left(x_{0}+t\right)=P_{x_{0}}(t)+R_{x_{0}}(t)$ where $P_{x_{0}}(t)$ is the usual polynomial in the development of $g$ we see that

$$
\left|R_{x_{0}}(t)\right|=\left.\frac{1}{(k-1) !}\left|\int_{0}^{|t|}(|t|-u)^{k-1} \omega_{x_{0}, \sigma}(u \sigma)\right| u\right|^{1-\beta} d u \mid
$$

where $\sigma=t /|t|$. Hence

$$
\begin{aligned}
\int_{E_{n}} \frac{\left|R_{x_{0}}(t)\right|^{2}}{|t|^{n+2 \alpha}} d t & =\int_{\Sigma} d \sigma \int_{0}^{\infty} \frac{\left|R_{x_{0}}(r \sigma)\right|^{2}}{r^{1+2 \alpha}} d r \\
& \leqq C \int_{\Sigma} d \sigma \int_{0}^{\infty}\left\{\int_{0}^{r} r^{k-\beta}\left|\omega_{x_{0}, \sigma}(u \sigma)\right| d u\right\}^{2} \frac{d r}{r^{1+2 \alpha}} \\
& =C \int_{\Sigma} d \sigma \int_{0}^{\infty}\left\{\frac{1}{r} \int_{0}^{r}\left|\omega_{x_{0}, \sigma}(u \sigma)\right| d u\right\}^{2} \frac{d r}{r}
\end{aligned}
$$


After an application of Hölder's inequality and a change of order of integration of the inner two integrals this is less than or equal to a constant times

$$
\int_{\Sigma} d \sigma \int_{0}^{\infty}\left|\omega_{x_{0}, \sigma}(u \sigma)\right|^{2}\left\{\int_{u}^{\infty} \frac{d r}{r^{2}}\right\} d u=\int_{E_{n}} \frac{\left|\omega_{x_{0}, \sigma}(t)\right|^{2}}{t^{n}} d t<\infty
$$

For the case $2 \leqq p<\infty$ let $A$ be an upper bound for $\omega_{x_{0}}(t)$. Then one has

$$
\int_{E_{n}} \frac{\left|\omega_{x_{0}}(t)\right|^{p}}{|t|^{n}} d t \leqq \frac{A^{p}}{A^{2}} \int_{E_{n}} \frac{\left|\omega_{x_{0}}(t)\right|^{2}}{|t|^{n}} d t<\infty .
$$

Lemma 3. Suppose that $f \in L^{2}\left(E_{n}\right)$ and has finite support, and that $F(x)=f_{\beta}(x)$, $0<\beta<1$. Then

$$
\frac{1}{A_{\beta}} \int_{|t| \geqq \varepsilon} \frac{F(x+t)-F(x)}{|t|^{n+\beta}} d t
$$

converges to $f(x)$ in the $L^{2}$ norm as $\varepsilon \rightarrow 0$ for a suitable choice of $A_{\beta} . A_{\beta}$ is a nonzero constant depending only on $\beta$ and $n$.

Proof. The Fourier transform of $F(x+t)-F(x)$ is $B_{\beta} f^{\wedge}(x)\left\{e^{i\langle x, t\rangle}-1\right\}|x|^{-\beta}$ where $B_{\beta}$ is the constant such that $\left\{1 /|x|^{n-\beta}\right\}^{\wedge}=B_{\beta}|x|^{-\beta}$. Therefore the Fourier transform of $\int_{|t| \geqq \varepsilon}\left((F(x+t)-F(x)) /|t|^{n+\beta}\right) d t=f^{\wedge}(x) M_{\varepsilon}(x)$ where

$$
M_{\varepsilon}(x)=B_{\beta}|x|^{-\beta} \int_{|t| \geqq \varepsilon} \frac{e^{i\langle x, t\rangle}-1}{|t|^{n+\beta}} d t .
$$

It is easy to see that $M_{\varepsilon}(x)$ is bounded in $x$ and $\varepsilon$ uniformly for $x \neq 0$ and that the limit of $M_{\varepsilon}(x)$ as $\varepsilon \rightarrow 0$ is nonzero. An application of Plancherel's theorem gives the required result.

Lemma 4. (See $[1, p .184]$.) Let $P$ be a closed set and $U=\{x: d(x, P)<1\}$. There exists a covering of $U-P$ by nonoverlapping cubes $K$ with the property that diam $K$ $\leqq d(P, K) \leqq 3 \operatorname{diam} K . d(x, P)$ and $d(P, K)$ represent the distance from $x$ to $P$ and from $P$ to $K$ respectively and diam $K$ represents the diameter of the cube $K$.

Here the conclusion has been slightly altered from that of [1] but it is not essentially different in the proof.

Lemma 5. (See [6, p. 130].) Let $P$ be a closed set and $U$ be as above. Set $\Delta(x)$ $=d(x, P)$ for $x$ in $U$ and zero otherwise. Then for $\lambda>0$ we have

for almost all $x_{0} \in P$.

$$
\int \frac{\Delta^{\lambda}\left(x_{0}+t\right)}{|t|^{n+\lambda}} d t<\infty
$$

Lemma $5^{\prime}$. (See [1,p. 189].) Suppose that $\lambda>0$ and

$$
\frac{1}{h^{n}} \int_{|t|<h} H\left(x_{0}+t\right) d t \leqq A h^{\lambda}, \quad 0<h<\infty,
$$

for every $x_{0}$ in $P$. Then $\int\left(H\left(x_{0}+t\right) /|t|^{n+\lambda}\right) d t<\infty$ for almost all $x_{0}$ in $P$. 
Lemma $5 " .\left(\right.$ See $[6, p .131]$.) Let $K_{\mu}$ be the sets of the cover in Lemma 4. Then

$$
\sum_{\mu} \frac{\left(\operatorname{diam} K_{u}\right)^{n+\lambda}}{\left|x_{0}-x_{\mu}\right|^{n+\lambda}}<\infty \quad \text { for almost all } x_{0} \text { in } P .
$$

Here $x_{\mu}$ is a point of $P$ such that $d\left(x_{\mu}, K\right)=d(P, K)$ and $\lambda>0$.

Lemma 6. (See [1, p. 183].) Suppose $\alpha>0$, with $k<\alpha<k+1$, and that $h$ has the development $h\left(x_{0}+t\right)=\sum_{|j|=0}^{k} h_{j}\left(x_{0}\right) t^{j}+R_{x_{0}}(t)$ for each $x_{0}$ in $P$, with $\left(1 / \rho^{n}\right) \int_{|t| \leqq \rho}\left|R_{x_{0}}(t)\right|^{p} d t \leqq A \rho^{\alpha}$ for $0<\rho \leqq \delta$. Then with $x_{0}$ in $P$ and $x_{0}+t$ in $P$,

$$
h_{e}\left(x_{0}+t\right)=\sum_{|j|=0}^{k-|e|} \frac{t^{j}}{j !} h_{j+e}\left(x_{0}\right)+O\left(|t|^{\alpha+|e|}\right)
$$

for $|e|=0,1, \ldots, k ; O$ is uniform for $x_{0}, x_{0}+t$ in $P$, and $|t| \leqq \delta$.

Lemma 7. (See [1, p. 189].) Suppose that $F$ has a $k+1$ derivative in the $L^{2}$ sense on a set $E,|E|>0$, uniformly. Also assume $F$ has finite support. Then there exists a function $G \in C_{0}^{k+1}$ such that $F=G+H$ where $H\left(x_{0}\right)=0$ for $x_{0}$ in $E$ and

$$
\frac{1}{\rho^{n}} \int_{|t|<\rho}\left|H\left(x_{0}+t\right)\right|^{2} d t=o\left(|t|^{2 k+2}\right) \quad \text { for } x_{0} \text { in E uniformly. }
$$

3. In this section we show the conditions of Theorem 1 are sufficient for $f$ to have an $\alpha$ derivative almost everywhere in $E$. As mentioned earlier we will not do the $L^{p}$ case since it differs from the $L^{2}$ case in an unessential way. In view of Theorem A it is enough to show that $F=f_{\beta}$ satisfies condition $\Lambda_{k+1}^{*}$ and $N_{k+1}^{2}$ almost everywhere in $E$.

Suppose that $x_{0}$ in $E$ is the origin and that $f$ has support contained in the sphere $S_{a}=\{x:|x| \leqq a\}$. Let $\lambda(t)$ be a function which is infinitely differentiable and has support in the sphere $S_{b}=\{x:|x| \leqq b\}$ with $b>a$ and $\lambda(t)=1$ for $|t| \leqq a$. Hence $f(t)=\lambda(t) f(t)$. Let $P(t)$ be the polynomial in (1) and $R(t)$ be the remainder. Then $f(t)=\lambda(t) P(t)+\lambda(t) R(t)$. The integral $\int_{E_{n}} \lambda(t) P(t)|x-t|^{\beta-n} d t$ represents an infinitely differentiable function. Hence we can make the simplifying assumptions that $x_{0}$ is the origin and that $f(t)=R(t)$ satisfies the condition $\Lambda_{\alpha}^{2}$ at $x_{0}$ and has support in $S_{b}$. We also assume $k$ even since the case $k$ odd is similar. Assume $0<|h|<b / 2$. Then

$$
\begin{aligned}
\frac{1}{2}\{F(h)+F(-h)\} & =\int_{S_{b}} R(t) \frac{1}{2}\left\{|h-t|^{\beta-n}+|h+t|^{\beta-n}\right\} d t \\
& =\int_{S_{b}} \frac{R(t)+R(-t)}{2} \cdot|h-t|^{\beta-n} d t \\
& =\int_{S_{b}} \omega(t)|t|^{\alpha} \cdot|h-t|^{\beta-n} d t .
\end{aligned}
$$

We first show the last integral is the sum of a polynomial in $h$ of degree $\leqq k+1$ and a remainder which is $O\left(|h|^{k+1}\right)$ as $|h| \rightarrow 0$. 
Split this integral into the two integrals $\int_{|t| \leqq 2|h|}+\int_{2|h| \leqq|t| \leqq b}$. The first is $\leqq O\left(|h|^{\alpha}\right) \int_{|t|<2|h|}|h-t|^{\beta-n} d t \leqq O\left(|h|^{k+1}\right)$. For the second, expand $|h-t|^{\beta-n}$ and $|h+t|^{\beta-n}$ in their respective Taylor's developments to obtain

$$
\int_{2|h| \leqq|t| \leqq b} \frac{R(t)}{2}|t|^{\beta-n}\left\{P\left(\frac{h}{|t|}\right)+O\left(\left|\frac{h}{t}\right|^{k+2}\right)\right\} d t
$$

where $P$ is a polynomial of degree $\leqq k+1$ containing even terms only, i.e., a term of $P(x)$ is $a_{j} x^{j}$ where $|j|$ is even. Since $R(t)=O\left(|t|^{\alpha}\right)$

$$
\int_{2|h| \leqq|t| \leqq b} O\left(\left|\frac{h}{t}\right|^{k+2}\right) \frac{R(t)}{2} d t=O\left(|h|^{k+1}\right) .
$$

For $|j|=0,2,4, \ldots, \frac{1}{2} k$ we have

$$
\begin{aligned}
\int_{2|h| \leqq|t| \leqq b} & \frac{R(t)}{2}|t|^{\beta-n} a_{j} \frac{h^{j}}{|t|^{|j|}} d t \\
= & a_{j} h^{j} \int_{|t| \leqq b} \frac{R(t)}{2}|t|^{\beta-n-|j|} d t+a_{j} h^{j} \int_{|t|<2|h|} O\left(|t|^{\beta-n+-|j|}\right) d t \\
& =a_{j} h^{j} \int_{|t| \leqq b} \frac{R(t)}{2}|t|^{\beta-n-|j|} d t+O\left(|h|^{k+1}\right) .
\end{aligned}
$$

Collecting these results we see we have $F$ equal to a polynomial of degree $\leqq k$ plus a term which is $O\left(|h|^{k+1}\right)$.

Now it remains to show that if $\eta(t)|t|^{k+1}=F(t)-P(t)$, where $P(t)$ is the polynomial obtained in the above argument, then

$$
\int_{|t| \leqq \rho} \frac{\eta^{2}(t)}{|t|^{n}} d t<\infty
$$

for some $\rho>0$. This will be accomplished if we show $\int_{|t| \leqq_{\rho}}\left(\eta_{i}^{2}(t) /|t|^{n}\right) d t<\infty$, $i=1,2,3$, where

$$
\begin{aligned}
& \eta_{1}(t)=|t|^{-(k+1)} \int_{|h| \leqq 2|t|} R(h)|h-t|^{\beta-n} d h \\
& \eta_{2}(t)=|t| \int_{2|t| \leqq|h| \leqq b} R(h)|h|^{-n-\alpha-1} d h \\
& \eta_{3}(t)=|t|^{-(k+1)+|j|} \int_{|h| \leqq 2|t|} R(h)|h|^{\beta-n-|j|} d h
\end{aligned}
$$

where $|j|=0,2,4, \ldots, k$.

In each of these cases a similar argument is used. We will do the argument in full for the case of $\eta_{1}$. Let $g(t)$ be a function such that

$$
\int_{|t| \leqq \rho} g^{2}(t) \frac{d t}{|t|^{2(\alpha+\beta)+n}}=1
$$


and assume that $\int_{|h| \leqq 2 \rho}\left(R^{2}(h) /|h|^{2 \alpha+n}\right) d h<\infty$. If we can show

$$
\int_{|t| \leqq \rho} g(t) \int_{|h| \leqq 2|t|} R(h)|h-t|^{\beta-n} d h \frac{d t}{|t|^{2(\alpha+\beta)+n}}
$$

is finite, this will prove

$$
\int_{|t| \leqq \rho}\left\{\int_{|h| \leqq 2|t|} R(h)|h-t|^{\beta-n} d h\right\}^{2} \frac{d t}{|t|^{2(\alpha+\beta)+n}}
$$

is finite as required. Rewriting the above using the notation that $t=|t| \sigma$ where $\sigma$ is the unit vector $t|t|^{-1}$ and $\tau=h|t|^{-1}$ we obtain

$$
\begin{aligned}
& \int_{0 \leqq|t| \leqq \rho} g(t) \frac{d t}{|t|^{2(\alpha+\beta)+n}} \int_{|\tau| \leqq 2} \frac{|t|^{\beta} R(|t| \tau)}{|\tau-\sigma|^{n-\beta}} d \tau \\
& \quad=\int_{0 \leqq|t| \leqq \rho ;|\tau| \leqq 2} \frac{g(t)}{|t|^{\alpha+\beta}} \cdot \frac{R(|t| \tau)}{|t|^{\alpha}} \frac{d t}{|t|^{n}} \cdot \frac{d \tau}{|\tau-\sigma|^{n-\beta}} .
\end{aligned}
$$

Applying Hölder's inequality with the measure $\left(d t /|t|^{n}\right) \cdot\left(d \tau /|\tau-\sigma|^{n-\beta}\right)$ this is

$$
\begin{aligned}
\leqq\left\{\int_{0 \leqq|t| \leqq \rho ;|\tau| \leqq 2} \frac{g^{2}(t)}{|t|^{2(\alpha+\beta)}} \frac{d t}{|t|^{n}}\right. & \left.\frac{d \tau}{|\tau-\sigma|^{n-\beta}}\right\}^{1 / 2} \\
& \times\left\{\int_{0 \leqq|t| \leqq \rho ;|\tau| \leqq 2} \frac{R^{2}(|t| \tau)}{|t|^{2 \alpha}} \frac{d t}{|t|^{n}} \frac{d \tau}{|\tau-\sigma|^{n-\beta}}\right\}^{1 / 2} .
\end{aligned}
$$

The square of the first integral in this product is

$$
\leqq \int_{0 \leqq|t| \leqq \rho} g^{2}(t) \frac{d t}{|t|^{2(\alpha+\beta)+n+\beta}} \int_{|h| \leqq 2|t|} \frac{d h}{|h-t|^{n-\beta}} .
$$

The inner integral is $\leqq C|t|^{\beta}$ and hence the first term in the product is finite. The square of the second term is equal to

$$
\begin{aligned}
\int_{|t| \leqq \rho} \int_{|h| \leqq 2|t|} & \frac{R^{2}(h)}{|h|^{2 \alpha+n}} \frac{|\tau|^{2 \alpha+n-\beta}|h|^{\beta}}{|h-t|^{n-\beta}} d h d t \\
& \leqq(2 \rho)^{\beta}(2)^{2 \alpha+n-\beta} \int_{|t| \leqq \rho} \int_{|h| \leqq 2 \rho} \frac{R^{2}(h)}{|h|^{2 \alpha+n}} d h \frac{d t}{|h-t|^{n-\beta}} \\
& \leqq(2 \rho)^{\beta}(2)^{2 \alpha+n-\beta} \int_{|h| \leqq 2 \rho} \int_{|t| \leqq 3 \rho} \frac{R^{2}(h)}{|h|^{2 \alpha+n}} d h \frac{d t}{|t|^{n-\beta}}<\infty
\end{aligned}
$$

4. We consider the necessity of the conditions of Theorem 1 and Theorem 2 from this point on.

The assumption $\left(1 / \rho^{n}\right) \int_{|t| \leqq \rho}\left|f\left(x_{0}+t\right)-P_{x_{0}}(t)\right|^{p} d t=O\left(\rho^{p \alpha}\right), 2 \leqq p<\infty$, clearly implies $f$ is locally integrable to the $p$ th power. Thus we may modify $f$ to have finite support and to be in $L^{p}$. In addition, we may limit our consideration to a closed set $P \subset E$, where $|E-P|<\varepsilon$ and $\varepsilon>0$ is arbitrary, on which $f_{\beta}=F$ has a $k+1$ derivative in the $L^{p}$ sense uniformly and satisfies the condition $\Lambda_{\alpha}^{p}$ uniformly there. 
By Lemma 7 we may write $F(x)=G(x)+H(x)$ where $G(x) \in C_{0}^{k+1}$ and $G_{j}\left(x_{0}\right)$ $=F_{j}\left(x_{0}\right)$ represents the $j$ th coefficient of the polynomial in (1). Also we know that $H=F-G$ is zero on $P$ and because of the uniform differentiability of $F$ and the fact that $H$ has compact support we have

$$
\int_{|t| \leqq \rho}\left|H\left(x_{0}+t\right)\right|^{p} d t \leqq \text { constant } \rho^{p(k+1)+n}, \quad 0<\rho<\infty,
$$

for $x_{0}$ in $P$.

Apply the inversion formula of Lemma 3 to $F$ to obtain

$$
f(x)=\lim _{\varepsilon \rightarrow 0} \frac{1}{A_{\beta}} \int_{|t| \geqq \varepsilon} \frac{F(x+t)-F(x)}{|t|^{n+\beta}} d t
$$

where the limit is taken in the $L^{2}$ norm. Also consider the function $g(x)=$ $\lim _{\varepsilon \rightarrow 0}\left(1 / A_{\beta}\right) \int_{|t| \geqq \varepsilon}\left(G(x+t)-G(x) /|t|^{n+\beta}\right) d t$. Since $G \in C_{0}^{k+1}$ this limit exists in the $L^{2}$ norm uniformly in $x$. Calculating $g(x)$ we find

$$
\begin{aligned}
g(x) & =\lim _{\varepsilon \rightarrow 0} \frac{1}{A_{\beta}} \int_{\Sigma} d \sigma \int_{\varepsilon}^{\infty} \frac{G(x+r \sigma)-G(x)}{r^{1+\beta}} d r \\
& =\lim _{\varepsilon \rightarrow 0} \frac{1}{A_{\beta}} \int_{\Sigma} d \sigma \int_{\varepsilon}^{\infty}\left\{\int_{0}^{r} G_{\sigma}(x+s \sigma) d s\right\} \frac{d r}{r^{1+\beta}} .
\end{aligned}
$$

After changing the order of integration of the inner integrals and letting $\varepsilon \rightarrow 0$ this becomes

$$
\frac{1}{\beta A_{\beta}} \int_{\Sigma} d \sigma \int_{0}^{\infty} G_{\sigma}(x+s \sigma) \frac{d s}{s^{\beta}}
$$

Note that $g(x)$ is a function which satisfies the hypothesis of Lemma 2.

Set $h(x)=f(x)-g(x)$. Then we have

$$
h(x)=\lim _{\varepsilon \rightarrow 0} \frac{1}{A_{\beta}} \int_{|t| \geqq \varepsilon} \frac{H(x+t)-H(x)}{|t|^{n+\beta}} d t
$$

in the $L^{2}$ norm. However $H(x)=0$ if $x$ in $P$ and the integral $\int_{E_{n}}\left(H(x+t) /|t|^{n+\beta}\right) d t$ converges for almost every $x$ in $P$ by Lemma $4^{\prime}$ and (6). By shrinking $P$ further we may assume that $h\left(x_{0}\right)=\left(1 / A_{\beta}\right) \int_{E_{n}}\left(H\left(x_{0}+t\right) /|t|^{n+\beta}\right) d t$ for all $x_{0}$ in $P$. Since $g$ satisfies the hypothesis of Lemma 2 , it satisfies the condition $\Lambda_{\alpha}$ uniformly and it satisfies the condition $N_{\alpha}^{p}, 2 \leqq p<\infty$, almost everywhere. Also $f$ satisfies the condition $\Lambda_{\alpha}$ uniformly in $P$; hence the problem of showing that $f$ satisfies the condition $N_{\alpha}^{p}$ almost everywhere in $P$ reduces to showing that $h$ satisfies the condition $N_{\alpha}^{p}$ almost everywhere in $P$. To do this we assume that $x_{0}$ is a point of density of $P$ at which the Lemmas 5, 5, $5^{\prime \prime}$ and 6 hold. We may assume that $x_{0}$ is the origin. Since the constant $A_{\beta}$ plays no essential role from this point on, we drop it.

Hence we need to show we can write

$$
h(x)=P(t)+\xi(t)|t|^{\alpha}
$$


where $P(t)=\sum_{|j|=0}^{k}\left(a_{j} t^{j} \mid j !\right)$ and $\int_{|t| \leqq 0}\left(|\xi(t)|^{p} /|t|^{n}\right) d t<\infty$. As before we will do the problem for the case $p=2$ since the case $2 \leqq p<\infty$ is similar. If $x$ is in $P$ we can write

$$
h(x)=\int_{E_{n}} \frac{H(t)}{|x-t|^{n+\beta}} d t=\int_{|t| \geqq 2|x|}+\int_{|t| \leqq 2 x}=S+T .
$$

If $|t| \geqq 2|x|$ then $|x-t|^{-(n+\beta)}=|t|^{-(n+\beta)}\left\{P_{t}(x)+R\right\}$ where $P_{t}(x)$ is the Taylor's development of $\left.|x /| t|-t /| t\right|^{-(n-\beta)}$ up to and including the $k$ th terms and $R$ is the remainder which is $O\left(|x / t|^{k+1}\right)$. Thus

where

$$
S=\sum_{|j|=0}^{k} A_{j} x^{j} \int_{E_{n}} \frac{H(t)}{|t|^{|j|}|t|^{n+\beta}} d t+R_{1}
$$

$$
R_{1}=\sum_{|j|=0}^{k} A_{j} x^{j} \int_{|t| \leqq 2|x|} \frac{H(t)}{|t|^{n+\beta+|j|}} d t+O\left(|x|^{k+1} \int_{|t| \geqq 2|x|} \frac{|H(t)|}{|t|^{k+1+\beta+n}} d t\right) .
$$

One can easily see that

$T=\int_{|t| \leqq 2|x|} \frac{|H(t)|}{|t-x|^{n+\beta}} d t \leqq \int_{|x / 2| \leqq|t| \leqq 2|x|} \frac{|H(t)|}{|t-x|^{n+\beta}} d t+$ constant $\int_{|t| \leqq 2|x|} \frac{|H(t)|}{|x|^{n+\beta}} d t$.

The first integral of $S$ is a polynomial of degree less than or equal to $k$. To show that $h$ satisfies the condition $N_{\alpha}^{2}$ it is enough to show that

$$
\int_{|x| \leqq \delta} \frac{I_{i}^{2}(x) d x}{|x|^{n}}<\infty
$$

$i=1,2,3,4$, for some $\delta>0$, where

$$
\begin{aligned}
& I_{1}(x)=|x|^{\beta} \int_{|t| \geqq 2|x|} \frac{|H(t)|}{|t|^{k+1+\beta+n}} d t, \\
& I_{2}(x)=|x|^{|j|-\alpha} \int_{|t| \leqq 2|x|} \frac{|H(t)|}{|t|^{n+\beta+|j|}} d t, \quad|j|=0,1, \ldots, k, \\
& I_{3}(x)=|x|^{-\alpha} \int_{|x| / 2 \leqq|t| \leqq 2|x|} \frac{|H(t)|}{|t-x|^{n+\beta}} d t, \\
& I_{4}(x)=|x|^{-(\alpha+\beta+n)} \int_{|t| \leqq 2|x|}|H(t)| d t .
\end{aligned}
$$

To do this we do the integration over the set of points which are in the set $P$ and later we consider the integration over the complement of $P$.

First consider the above integral with $i=1$. As in an earlier argument suppose that $g(x)$ is such that $\int_{|x| \leqq \delta}\left(g^{2}(x) /|x|^{n}\right) d x=1$. Then we must show $\int_{|x| \leqq \delta}\left(g(x) I_{1}(x) /|x|^{n}\right) d x<\infty$. This integral is equal to

$$
\begin{aligned}
& \int_{|x| \leqq \delta} \int_{|t| \geqq 2|x|} g(x)|x|^{\beta / 2} \frac{|x|^{\beta / 2}|H(t)|}{|t|^{k+1}} \frac{d t}{|t|^{n+\beta}} \frac{d x}{|x|^{n}} \\
& \leqq\left\{\int_{|x| \leqq \delta} \int_{|t| \geqq 2|x|} g^{2}(x)|x|^{\beta} \frac{d t}{|t|^{n+\beta}} \frac{d x}{|x|^{n}}\right\}^{1 / 2}\left\{\int_{|x| \leqq \delta} \int_{|t| \geqq 2|x|} \frac{|x|^{\beta} H^{2}(t)}{|t|^{2(k+1)}} \frac{d t}{|t|^{n+\beta}} \frac{d x}{|x|^{n}}\right\}^{1 / 2} .
\end{aligned}
$$


Changing the order of integration in the second integral of this product and integrating over all $x$ gives

$$
\int_{|t| \geqq 0} \int_{|x| \leqq t / 2}|x|^{\beta} \frac{H^{2}(t)}{|t|^{2(k+1)}} \frac{d x}{|x|^{n}} \frac{d t}{|t|^{n+\beta}} \leqq \text { constant } \int_{|t| \geqq 0} \frac{H^{2}(t)}{|t|^{2(k+1)+n}} d t<\infty .
$$

On the other hand, one can easily see the first term in the product is finite.

In a similar way one can obtain the required results in the cases for the integrals $I_{2}$ and $I_{4}$. For the remainder of this section we concentrate on $I_{3}$.

Let $U=\{x: d(x, P)<1\}$. Let $Q=U-P$ and let $K_{\mu}$ be the elements of the cover of $Q$ as given in Lemma 4. Let $x$ be in $P$ and consider the integral $\int_{t \in K_{\mu}}\left(|H(t)| /|x-t|^{n+\beta}\right) d t$. We have that, the diameter of $K=\operatorname{diam}\left(K_{\mu}\right)$, diam $\left(K_{\mu}\right)$ $\leqq d\left(P, K_{\mu}\right) \leqq 3 \operatorname{diam}\left(K_{\mu}\right)$. For each $K_{\mu}$ we let $a_{\mu}$ be the center of $K_{\mu}$, and $x_{\mu}$ be a point of $P$ such that $d\left(x_{\mu}, K_{\mu}\right)=d\left(P, K_{\mu}\right)$. Let $S_{\mu}$ be the smallest sphere containing $K_{\mu}$ with center at $x_{\mu}$.

Suppose that $d\left(x, x_{\mu}\right) \geqq 4 \operatorname{diam}\left(K_{\mu}\right)$. Then

$$
\int_{K_{\mu}} \frac{|H(t)|}{|x-t|^{n+\beta}} d t \leqq C\left|x-x_{\mu}\right|^{-(n+\beta)}\left\{\int_{S_{\mu}}|H(t)| d t\right\}
$$

since $|x-t| \geqq \frac{1}{4}\left|x-x_{\mu}\right|$. By (6) this is $\leqq C\left|x-x_{\mu}\right|^{-(n+\beta)} \operatorname{diam}\left(K_{\mu}\right)^{k+1+n}$ $=C\left|x-x_{\mu}\right|^{-(n+\beta)} \int_{K_{\mu}} \Delta^{k+1}(t) d t$ where $\Delta(t)$ is the distance of $t$ from $P$. Since $|x-t| \leqq 3\left|x-x_{\mu}\right|$ the last line is a constant times $\int_{K_{\mu}}\left(\Delta^{k+1}(t) /|x-t|^{n+\beta}\right) d t$. Suppose that $\left|x-x_{\mu}\right|<4 \operatorname{diam}\left(K_{\mu}\right)$. Then

$$
\int_{K_{\mu}} \frac{|H(t)|}{|x-t|^{n+\beta}} d t \leqq C \int_{K_{\mu}} \frac{|H(t)|}{\left|x_{\mu}-t\right|^{n+\beta}} d t
$$

since $|x-t| \geqq \operatorname{diam}\left(K_{\mu}\right)$ and $\left|x_{\mu}-t\right| \leqq 4 \operatorname{diam}\left(K_{\mu}\right)$. We have $\left|x_{\mu}-a_{\mu}\right| \leqq 4 \operatorname{diam}\left(K_{\mu}\right)$ $\leqq 4\left|x_{\mu}-t\right|$ and the above integral is

$$
\begin{aligned}
& \leqq C \int_{S_{\mu}} \frac{|H(t)|}{\left|x_{\mu}-a_{\mu}\right|^{n+\beta}} d t \leqq C\left|x_{\mu}-a_{\mu}\right|^{\alpha} \\
& \leqq C\left|x_{\mu}-a_{\mu}\right|^{-(n+\beta)} \int_{K_{\mu}} \Delta^{k+1}(t) d t .
\end{aligned}
$$

One can show $\left|x_{\mu}-a_{\mu}\right| \geqq|x-t| / 8$ for $t$ in $K_{\mu}$ so we find that

$$
\int_{K_{\mu}} \frac{|H(t)|}{|x-t|^{n+\beta}} d t \leqq C \int_{K_{\mu}} \frac{\Delta^{k+1}(t)}{|x-t|^{n+\beta}} d t \quad \text { for } x \text { in } P .
$$

Since the origin is a point of density of $P$ and $K_{u} \subset Q$, diam $\left(K_{\mu}\right)=o\left(\operatorname{diam} S_{\mu}(o)\right)$ as $\operatorname{diam}\left(S_{\mu}(o)\right) \rightarrow 0$ where $S_{\mu}(o)$ is the smallest sphere containing $K_{\mu}$ with center at the origin. Hence there is a $\delta>0$ such that for $\operatorname{diam}\left(S_{\mu}(0)\right)<2 \delta$, diam $\left(K_{\mu}\right)$ 
$<\operatorname{diam}\left(S_{\mu}(0)\right) / 6$. If we assume that $|x|<\delta$ then it is easy to see that if $E_{x}=$ $\{t:|x| / 2 \leqq|t| \leqq 2|x|\}$

$$
\begin{aligned}
\int_{|x| 2|\leqq| t|\leqq 2| x \mid} \frac{|H(t)|}{|x-t|^{n+\beta}} d t & \leqq \sum_{K_{\mu} \cap E_{x} \neq \varnothing} \int_{K_{\mu}} \frac{H(t)}{|x-t|^{n+\beta}} d t \leqq C \sum_{K_{\mu} \cap E_{x} \neq \varnothing} \int_{K_{\mu}} \frac{\Delta^{k+1}(t)}{|x-t|^{n+\beta}} d t \\
& \leqq C \int_{|x| 6|\leqq| t|\leqq 4| x \mid} \frac{\Delta^{k+1}(t)}{|x-t|^{n+\beta}} d t .
\end{aligned}
$$

If we choose $\delta<1 / 4$ the integration is over a region contained in $U$. Set $\Delta(t)=0$ if $t \notin U$. By applying the estimation technique used on the other integrals one can now show that $\int_{|x| \leqq \delta}\left(I_{3}^{2}(x) /|x|^{n}\right) d x<\infty$ after noting that $x$ in $P$ and, $\int\left(\Delta^{\lambda}(x) /|x|^{\lambda+n}\right) d x$ $<\infty$ for $\lambda>0$ by Lemma 5 , where one will need to use $\lambda=2(k+1)$.

5. To complete the demonstration that $h$ satisfies the condition $N_{\alpha}^{2}$ one must show that $\int_{Q ;|x| \leqq \delta}\left(\xi^{2}(x) /|x|^{n}\right) d x<\infty$. Since $\left\{K_{\mu}\right\}$ is a cover for $Q$ the finiteness of this will be proved if we show the two sums

$$
\sum_{\mu} \int_{K_{\mu}}\left[\rho(x)-\rho\left(x_{\mu}\right)\right]^{2} \frac{d x}{|x|^{2 \alpha+n}}
$$

and

$$
\sum_{\mu} \int_{K_{\mu}}\left[\rho\left(x_{\mu}\right)\right]^{2} \frac{d x}{|x|^{2 \alpha+n}}
$$

are finite, where $\xi(x)|x|^{\alpha}=\rho(x)$.

We consider (10) first. Since $x_{\mu}$ is a point of $P, \xi\left(x_{\mu}\right)$ is majorized by the sum of the integrals $I_{1}, I_{2}, I_{3}$ and $I_{4}$ with $x=x_{\mu}$. Since the origin is a point of density there is a $\delta>0$ such that for $K_{\mu} \subset\{x:|x|<\delta\}$ and $x$ in $K_{\mu},\left(\left|x_{\mu}\right| / 2\right) \leqq|x| \leqq 2\left|x_{\mu}\right|$ and hence in this case $(10)$ is majorized by a constant times

$$
\sum_{\mu} \int_{K_{\mu}} \frac{\xi^{2}\left(x_{\mu}\right)}{|x|^{n}} d x .
$$

One sees that, for $x$ in $K_{\mu}, I_{1}\left(x_{\mu}\right)$ is increased by a constant times

$$
|x|^{\beta} \int_{|t| \geqq|x|} \frac{|H(t)|}{|t|^{k+1+\beta+n}} d t
$$

Likewise $I_{2}\left(x_{\mu}\right)$ and $I_{4}\left(x_{\mu}\right)$ majorized by constant multiples of

$$
|x|^{|j|-\alpha} \int_{|t| \leqq 4|x|} \frac{|H(t)|}{|t|^{n+\beta+|j|}} d t, \quad|j|=0,1, \ldots, k
$$

and $|x|^{-(\alpha+\beta+n)} \int_{|t| \leqq 4|x|}|H(t)| d t$ respectively. The contribution of these integrals to (11) can be estimated in the same way as in the previous case. 
It remains to estimate the contribution of $I_{3}\left(x_{\mu}\right)$ to the convergence of (11). In view of (8) and the fact that $x_{\mu}$ is in $P$ we have (11) is finite if

$$
\begin{aligned}
\sum_{\mu} \int_{K_{\mu}} \frac{d x}{|x|^{n}}\left\{\left|x_{\mu}\right|^{-\alpha} \int_{\left|x_{\mu}\right| / 4 \leqq|t| \leqq 4\left|x_{\mu}\right|} \frac{|H(t)|}{\left|t-x_{\mu}\right|^{n+\beta}} d t\right\}^{2} & \leqq C \sum_{\mu} \int_{K_{\mu}} \frac{d x}{|x|^{n+\alpha}} \int_{|x| / 16 \leqq|t| \leqq 16|x|} \frac{\Delta^{k+1}(t)}{\left|t-x_{\mu}\right|^{n+\beta}} d t
\end{aligned}
$$

is finite. The last inequality follows by a use of the inequalities $\Delta(t) \leqq\left|t-x_{\mu}\right|$ and $\left|x_{\mu}\right| / 2 \leqq|x| \leqq 2\left|x_{\mu}\right|$. The limits of integration can be refined.

Let $S_{\mu}^{\prime}$ be the sphere with center at $x_{\mu}$ and radius twice that of $S_{\mu}$. It is easy to see that $\int_{S_{\mu}^{\prime}}\left(\Delta^{k+1}(t) /\left|x_{\mu}-t\right|^{n+\beta}\right) d t \leqq C\left[\operatorname{diam}\left(K_{u}\right)\right]^{\alpha}$ by noting that $\Delta^{k+1}(t) \leqq\left|x_{\mu}-t\right|$ and that $\operatorname{diam}\left(S_{\mu}\right) \leqq C \operatorname{diam} K_{\mu}$. Also, since $|x| \geqq\left|x_{\mu}\right| / 16$, one can see that

$$
\int_{K_{\mu}} \frac{d x}{|x|^{n+\alpha}} \int_{S_{\mu}^{\prime}} \frac{\Delta^{k+1}(t)}{\left|x_{\mu}-t\right|^{n+\beta}} d t
$$

is majorized by a constant times [diam $\left.K_{\mu}\right]^{n+\alpha} \cdot\left|x_{\mu}\right|^{-(n+\alpha)}$. Hence by the Lemma $5^{\prime \prime}$ the contribution of (11) on the $S_{\mu}^{\prime}$ is finite and we only need to consider the contribution over the $t$ with $|x| / 16 \leqq|t| \leqq 16|x|$ outside the $S_{\mu}^{\prime}$. For such $t,\left|t-x_{\mu}\right|$ $\geqq \frac{1}{2}|t-x|$ since $x \in K_{\mu} \subset S_{\mu}$. Let $\psi_{Q}$ be the characteristic function of $Q$ and let $\lambda_{x}(t)$ be equal to zero if $t \in S_{\mu}^{\prime}$ and $x \in K_{\mu}$, and let $\lambda_{x}(t)$ be one otherwise. Then the part of (11) that remains to be considered is a constant multiple of

$$
\begin{aligned}
\int_{Q} \frac{d x}{|x|^{n+\alpha}}\left\{\int_{|x| / 16 \leqq|t| \leqq 16|x|} \frac{\Delta^{k+1}(t) \lambda_{x}(t)}{|t-x|^{n+\beta}} d t\right\} \\
\leqq C \int_{E_{n}} \frac{\Delta^{k+1}(t)}{|t|^{n+\alpha}} d t \int_{|t| / 16 \leqq|x| \leqq 16|t|} \frac{\psi_{Q}(x) \lambda_{x}(t)}{|t-x|^{n+\beta}} d x
\end{aligned}
$$

Now the inner integral is taken over the exterior of $Q$ to the sphere $S_{\mu}^{\prime}$ which contains $x$ in $K_{u}$, i.e., it is

$$
\leqq C \int_{2|x-t| \geqq \Delta(t)} \frac{d x}{|t-x|^{n+\beta}} \leqq C \Delta^{-\beta}(t) .
$$

Combining this with the above we have that (12) is dominated by

$$
C \int_{|t| \leqq \infty} \frac{\Delta^{k+1-\beta}(t)}{|t|^{n+\beta}} d t=C \int \frac{\Delta^{\alpha}(t)}{|t|^{n+\alpha}} d t
$$

This is finite by Lemma 5 .

It now remains to show that (9) is finite. To do this recall that $h$ satisfies the condition $\Lambda_{\alpha}^{2}$ uniformly in the set $P$, i.e., we can write $f\left(x_{0}+t\right)=P_{x_{0}}(t)+\rho_{x_{0}}(t)$ where $P_{x_{0}}(t)$ is a polynomial of degree $\leqq k$ and $\int_{|t| \leqq \rho}\left|\rho_{x_{0}}(t)\right|^{2} d t=O\left(\rho^{2 \alpha+n}\right)$, as $\rho \rightarrow 0$, uniformly for $x_{0}$ in $P$. We can apply Lemma 6 to $h$ to see that for $x_{0}$ the origin we have $h(x)=P(x)+\rho(x)$ as in (7) and if we write $x_{\mu}+t=x$ we have $h(x)=\sum_{|j|=0}^{k}\left(h_{j}\left(x_{\mu}\right) t^{j} \mid j !\right)+\rho_{x_{\mu}}\left(x-x_{\mu}\right)$. For $x$ in $P(\partial / \partial x)^{j} P(x)=h_{j}(x)$ and hence we 
have $h_{j}\left(x_{\mu}\right)-(\partial / \partial x)^{j} P\left(x_{\mu}\right)=O\left(\left|x_{\mu}\right|^{\alpha-|j|}\right)$. Also we have that $P(x)=\sum_{|j|=0}^{k}(\partial / \partial x)^{j}$ $\times P\left(x_{\mu}\right)\left(x-x_{\mu}\right)^{j} / j$ !. Combining all these we can write

$$
\begin{aligned}
\rho(x)-\rho\left(x_{\mu}\right) & =h(x)-h\left(x_{\mu}\right)-\left\{P(x)-P\left(x_{\mu}\right)\right\} \\
& =\sum_{|j|=1}^{k} \frac{h_{j}\left(x_{\mu}\right)}{j !} t^{j}+\rho_{x_{\mu}}\left(x-x_{\mu}\right)-\sum_{|j|=1}^{k} \frac{p_{j}\left(x_{\mu}\right)\left(x-x_{\mu}\right)^{j}}{j !} .
\end{aligned}
$$

Hence

$$
\left|\rho(x)-\rho\left(x_{\mu}\right)\right| \leqq \rho_{x_{\mu}}\left(x-x_{\mu}\right)+O\left(\sum_{|j|=1}^{k}\left|x_{\mu}\right|^{\alpha-|j|}\left|x-x_{\mu}\right|^{|j|}\right) .
$$

Using this to estimate (9) we see that with the definition of $h$ in $\Lambda_{\alpha}^{2}$

$$
\sum_{\mu} \frac{\left[\rho(x)-\rho\left(x_{u}\right)\right]^{2}}{|x|^{2 \alpha+n}} d x \leqq C \sum_{\mu} \frac{\left[\operatorname{diam}\left(K_{\mu}\right)\right]^{n+2 \alpha}}{\left|x_{\mu}\right|^{n+2 \alpha}}+C \sum_{\mu} \frac{\left[\operatorname{diam}\left(K_{\mu}\right)\right]^{n+2}}{\left|x_{\mu}\right|^{n+2}} .
$$

The last inequalities are demonstrated by recalling the inequalities involving $x$ and $x_{\mu}$ where $x$ is in $K_{\mu}$ ( (9) is finite by Lemma $5^{\prime \prime}$.

As a final remark we point out that the condition $N_{\alpha}^{p}$ easily implies the condition $\Lambda_{\alpha}^{p}$ which is enough to complete the proof of Theorem 2 and Theorem 2'.

\section{BIBLIOGRAPHY}

1. A. P. Calderón and A. Zygmund, Local properties of solutions of elliptic partial differential equations, Studia Math. 20 (1961), 171-225.

2. G. H. Hardy, J. E. Littlewood and G. Pólya, Inequalities, Cambridge Univ. Press, Cambridge, 1952.

3. E. M. Stein, Singular integrals, harmonic functions, and differentiability properties of functions of several variables, Proc. Sympos. Pure Math., Vol. 10, Amer. Math. Soc., Providence, R. I., 1968, pp. 316-335.

4. E. M. Stein and A. Zygmund, On the differentiability of functions, Studia Math. 23 (1964), 247-283.

5. - On the fractional differentiability of functions, Proc. London Math. Soc. 14A (1965), 249-264.

6. A. Zygmund, Trigonometric series, 2nd ed., Chelsea, New York, 1959.

DePaul University,

Chicago, Illinois 